\title{
COMMISSIONING PLAN FOR A HIGH-CURRENT PROTON LINAC CONF-970503-- 266
}

K. C. D. Chan, R. L. Barber, R. W. Garnett, J. D. Gilpatrick, K. F. Johnson, G. P. Lawrence, S. Nath, A. Regan, L. Rybarcyk, R. E. Shafer, H. V. Smith, T. P. Wangler, and L. M. Young, Los Alamos National Laboratory, Los Alamos, NM 87545, L. W. Funk, Westinghouse Savannah River Company, Aiken, SC 29808 and K. Crandall, Amparo Corporation, Santa Fe, NM 87504.

\section{Abstract}

High-power proton linacs $(\mathrm{E}>500 \mathrm{MeV})$ are potentially useful for transmutation applications, such as the production of tritium. In production applications, high availability is essential. Achieving high availability requires an accelerator design that simplifies maintenance and accommodates commissioning procedures designed to minimize tune-up time. These are worthwhile goals for any accelerator, but the very high beam powers $(170 \mathrm{MW})$ and heavy beam loading of the Accelerator Production of Tritium (APT) [1] linac introduce significant new challenges. This paper will describe the commissioning plan, as developed to date.

\section{INTRODUCTION}

The APT accelerator is an integrated normal conducting - superconducting (NC/SC) linac [2]. It has a final beam energy of $1700 \mathrm{MeV}$ and a $\mathrm{CW}$ beam current of $100 \mathrm{~mA}$. It starts with a $75-\mathrm{kV}$ injector. Acceleration up to $217 \mathrm{MeV}$ is provided by a RFQ accelerator and a coupled-cavity NC linac. From 217 to $1700 \mathrm{MeV}$, acceleration is provided by two sections of SC linac: the medium $-\beta$ and high- $\beta$ linac sections.

The APT accelerator must demonstrate high reliability and availability. To achieve this, designers must know how the accelerator will be commissioned and operated. The design, commissioning plan and operating plan must be developed in concert to assure that the required beam instrumentation, controls and access will be available.

The goal of commissioning is to safely, quickly and reproducibly bring the beam to full current and energy with minimum beam loss, to verify that all systems are working correctly, and to develop a physics model of the beam dynamics that will permit computer-aided automatic beam tuning.

A good physics model and automatic beam tuning are important for achieving high availability. The most difficult source of 'emittance noise' to control is the degree of the neutralization [3] in the LEBT, upstream of the RFQ [4]. RFQs have been observed to be effective 'emittance filters'. Because RF accelerating systems downstream of the RFQ are well understood and known to be capable of consistent performance with good commissioning protocols, automatic beam tuning is

* Work supported by the US Department of Energy believed to be achievable.

$$
-1
$$

\section{IMPORTANT ELEMENTS 1997 COMMISSIONING

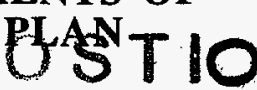

The commissioning plan has been laid out usijg recent commissioning experience from the FNAL linac upgrade and CEBAF. It is also based on the operatung experience at the Los Alamos Neutron Scattering Center (LANSCE).

The 170-MW APT linac can quickly activate or destroy components. It is therefore essential to approach full power with a low averaged power pulsed beam. Table 1 shows the different beam formats that will be used. A pilot beam is the lowest power beam used for equipment checkout when commissioning begins. Its minimum power is limited by the response of the diagnostics and the pulse rate of the ion source. A calibration beam is used for calibrating the amplitude and phase of the accelerating structures. Its power level is defined by the minimum beam power required by each calibration method. A tuneup beam brings the peak current to $100 \mathrm{~mA}$ without exceeding the design power of the full-energy beam stop at the end of the linac (Fig, 1) of $3.4 \mathrm{MW}$. The average power level of the pilot and calibration beam are comparable to that used for LANSCE tuneup. The LANSCE tuneup beam is a $480-\mathrm{W}$ pulsed beam of $4 \mathrm{~Hz}$, $1-\mathrm{mA}$ peak, and $150-\mu \mathrm{m}$ long.

Table. 1 List of Pulsed-Beam Formats

\begin{tabular}{|l|c|c|c|c|}
\hline $\begin{array}{c}\text { Beam } \\
\text { Types }\end{array}$ & $\begin{array}{c}\text { Repetition } \\
\text { Rate } \\
(\mathrm{Hz})\end{array}$ & $\begin{array}{c}\text { Max. Peak } \\
\text { Current } \\
(\mathrm{mA})\end{array}$ & $\begin{array}{c}\text { Pulse Length } \\
(\mathbf{m s})\end{array}$ & $\begin{array}{c}\text { Maximum } \\
\text { average } \\
\text { Power }\end{array}$ \\
\hline Pilot & 1 & 1 & 0.1 & $170 \mathrm{~W}$ \\
\hline Calibration & 1 & 10 & 1 & $17 \mathrm{~kW}$ \\
\hline Tuneup & 10 & 100 & 1 & $1.7 \mathrm{MW}$ \\
\hline APT & (CW) & 100 & $(\mathrm{CW})$ & $170 \mathrm{MW}$ \\
\hline
\end{tabular}

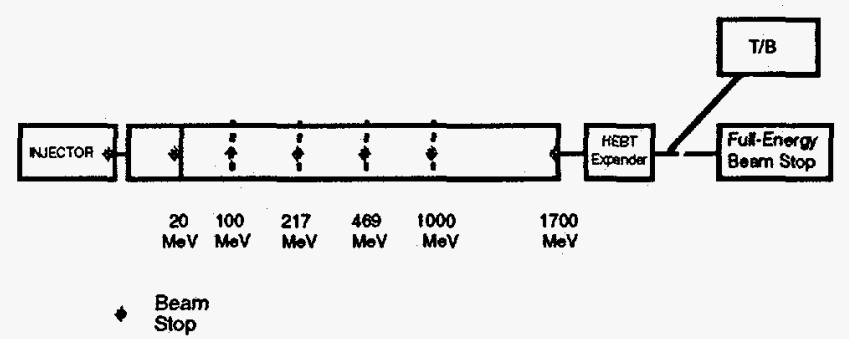

Fig. 1 Schematics of APT Linac for Commissioning

Different beam stops (Table 2 and Fig. 1) will be required at each commissioning step. A full power beam stop will be installed at the Injector for beam setup and 


\section{DISCLAIMER}

This report was prepared as an account of work sponsored by an agency of the United States Government. Neither the United States Government nor any agency thereof, nor any of their employees, make any warranty, express or implied, or assumes any legal liability or responsibility for the accuracy, completeness, or usefulness of any information, apparatus, product, or process disclosed, or represents that its use would not infringe privately owned rights. Reference herein to any specific commercial product, process, or service by trade name, trademark, manufacturer, or otherwise does not necessarily constitute or imply its endorsement, recommendation, or favoring by the United States Goverament or any agency thereof. The views and opinions of authors expressed herein do not necessarily state or reflect those of the United States Government or any agency thereof. 


\section{DISCLAMMER}

Portions of this document may be illegible in electronic image produets. Images are produced from the best available original document. 
beam abort. A full-power beam stop is required at the 20$\mathrm{MeV}$ point because the beam dynamic up to this energy is highly space-charge dominated. Good beam quality at this point is critical to the beam quality for the rest of the linac. The short APT schedule calls for linac installation by sections. Commissioning of a section will take place while later sections are being installed. To accomplish this, a low-average power beam stop that can be moved to the end of a section is needed. The radiation safety aspect of such a beam stop is presently under study. There is a full-energy, $2 \%$ full averaged power, beam stop at the end of the linac. The target/blanket will be used as a beam stop for CW beam tuning at power level from 2 to $100 \%$ of the full beam power.

Table. 2 List of APT Beam Stops

\begin{tabular}{|c|c|c|c|c|}
\hline Location & $\begin{array}{c}\text { CW or } \\
\text { Pulsed }\end{array}$ & $\begin{array}{c}\text { Average } \\
\text { Power }\end{array}$ & $\begin{array}{c}\text { Peak } \\
\text { Current } \\
(\mathrm{mA})\end{array}$ & $\begin{array}{c}\text { Energy } \\
(\mathrm{MeV})\end{array}$ \\
\hline Injector & $\begin{array}{c}\mathrm{CW} \text { and } \\
\text { Pulsed }\end{array}$ & $10 \mathrm{~kW}$ & 130 & 0.075 \\
\hline $20-\mathrm{MeV}$ & $\begin{array}{c}\text { CW and } \\
\text { Pulsed }\end{array}$ & $2 \mathrm{MW}$ & 100 & 20 \\
\hline $\begin{array}{c}\text { End of } \\
\text { Sections }\end{array}$ & Pulsed & $17 \mathrm{~kW}$ & 10 & 1700 \\
\hline $\begin{array}{c}\text { End of } \\
\text { Linac }\end{array}$ & Pulsed & $1.7 \mathrm{MW}$ & 100 & 1700 \\
\hline
\end{tabular}

Measurements using beam diagnostic instrumentation will be used to develop the physics model for automatic tuning. A list of measurements and measurement methods is given in Table 3. A description of the APT beam diagnostics can be found in Ref. [5].

\section{COMMISSIONING STEPS}

The APT-linac commissioning plan has the following steps (Fig. 1):

1. Installation of linac sections. Sections of linac are

Table 3 Diagnostics for Beam Measurements

\begin{tabular}{|l|l|}
\hline Measurements & \multicolumn{1}{|c|}{ Method } \\
\hline Current & Multi-core toroidal \\
\hline CW & Single-core toroidal \\
\hline Pulsed & $\begin{array}{l}\text { Summed BPM } \\
\text { signals }\end{array}$ \\
\hline Peak current & Ion chamber \\
\hline Beam loss & Ion chamber \\
\hline Fast Protect & \\
\hline Beam centroids & BPM \\
\hline Position & $\Delta$ between BPMs \\
\hline Angle & Capacity probe \\
\hline Phase & Time of flight \\
\hline Energy & \\
\hline Beam width & $\begin{array}{l}\text { Gas Fluorescence or } \\
\text { Flying wire }\end{array}$ \\
\hline Transverse & Dispersive Magnet \\
\hline Energy & Image Current Probe \\
\hline Phase & Scraper \\
\hline Halo &
\end{tabular}

defined temporarily at beam energies: $75 \mathrm{kV}, 20,100$, $217,469,1000$, and $1700 \mathrm{MeV}$. A section will be installed. Equipment will be checked without beam and with the pilot beam. Accelerating structures will be calibrated using the calibration beam.

2. The first $20 \mathrm{MeV}$ will be commissioned to full current using procedures developed in the Low-Energy Demonstration Accelerator.

3. At the completion of step 1 and 2 , the pulsed tuneup beam will be tuned to design peak current at the full energy beam stop.

4. A CW beam will be tuned to the Target/Blanket System. The current will increase from $2 \%$ to $100 \%$ in steps.

5. Linac physics-model study and beam-loss minimization will take place.

Methods used in these steps are described in the following subsections.

\section{$2.1 \quad$ Steering}

A pilot beam will be used. The principal diagnostics that will be used to establish alignment are the beam position monitors (BPM), of which about 210 will be permanently installed.

Several steering strategies have been investigated [6], of which two are particularly promising: dipole pair and least squares steering. Both are equally effective in keeping the beam centered in the beam channel, but the latter, although it requires somewhat more power supplies, reduces the demands on the steering magnets.

This stage will be carried out with the accelerating cavities detuned to minimize their interaction with the beam. It will be done by linac sections

\subsection{Cavity Field Amplitude and Phase for NC Structures}

In the normal conducting systems, over the physical region between two beam monitors, a series of phase scans and delta-t measurements [7] will establish the field phase and amplitude required to achieve design longitudinal beam dynamics. The minimum length for this process is one RF 'supermodule' [8]. A plot of the beam energy and output phases as a function of the RF amplitude and phase will be compared with simulation to determine the amplitude and phase of the RF cavity field.

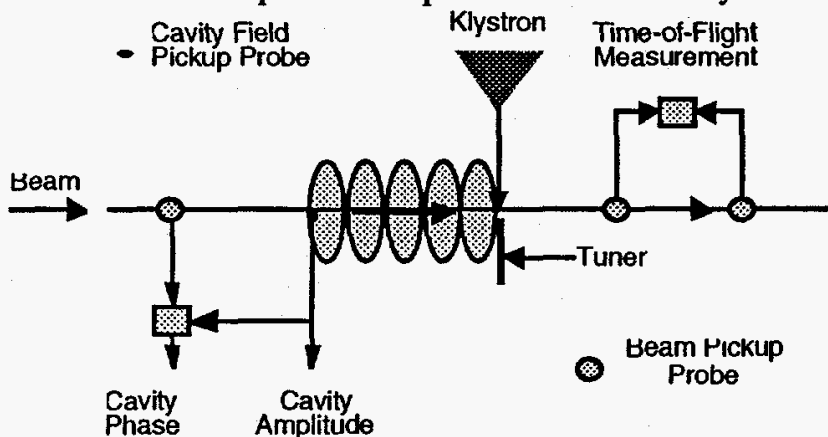

Fig. 2 A schematic of the setup for calibrating phase and amplitude of SC cavities 


\subsection{Cavity Field Amplitude and Phase for SC Cavities}

The APT SC linac can operate with some cavities idle. This flexibility of operation requires phase calibration of cavity independent of the number of cavities operating upstream of the cavity being calibrated. Figure 2 shows a schematic of the setup used for calibrating the amplitude and phase of an SC cavity. We plan to use capacitive pickups immediately upstream of the cryomodule to provide the beam arrival phase. The phase of the cavity field will reference to this arrival phase. By directly referencing to the beam arrival phase, the dependence on the number of operating cavities of cavity phase calibration is minimized. The first step of the calibration procedure is to establish a RF field in the cavity using the klystron while the cavity is resonant in frequency. A calibration beam will then be injected. The calibration of the phase calibration is achieved by adjusting the phase of the RF field until minimum beam induced phase changes of the cavity field is reached. AT that time, the RF field is orthogonal to the beam in phase.

Calibrating field amplitude is conceptually more straightforward. The RF field amplitude is measured by field probes that are weakly coupled to the RF field. The field probe is calibrated to $\pm 10 \%$ by measuring RF powers before installations of the cavity. Final calibration of the probe will be achieved by measuring the change in beam energy using the time-of-flight method using beam pickups along the accelerator.

\subsection{Tuneup of Full Peak and Average Current}

After the accelerating structures have been calibrated in RF phases and amplitudes, beam tuneup of the linac will star. The peak current will be increased to $100 \mathrm{~mA}$. The repetition rate will be increased but keeping the average beam power below the design power of the beam stop at the end of the linac. Once acceptable performance has been achieved at this power level, further increases in beam power will have to take place with the switchyard configured to send the beam to the Target/Blanket.

At the beginning of the tuneup to the Target/Blanket, the peak current has to be reduced to less than $2 \mathrm{~mA}$. The reduction of beam current will not affect the component settings along the linac because the linac has been designed for current independent matches [9].

Throughout the process, accelerator performance will be compared to simulations. Beam transmission, transverse beam position, beam energy, transverse and longitudinal beam sizes, transverse match, and beam halo will be measured. Finally, a physics model will be confirmed using the data obtained. Algorithms for minimizing beam loss will be tested.

\section{SUMMARY}

Work is underway to develop a commissioning plan for the integrated normal/superconducting linac of the APT, which developed in concert with the linac design and operation plan will lead to high availability. To date, commissioning steps have been laid out. Pulsed-beam formats, beam stops, and diagnostics required have been specified. Calibration methods of phase and amplitude of accelerating structures are being investigated.

\section{REFERENCES}

[1] Paul J. Lisowski, 'The Accelerator Production of Tritium (APT) Project', these proceedings.

[2] G. Lawrence and T. Wangler, 'Integrated Normalconducting/Superconducting High Power Proton Linac for APT", these proceedings.

[3] R. Ferdinand, J. Sherman, R. R. Stevens, Jr., and T. Zaugg, 'SpaceCharge Neutralization Measurement of a $75 \mathrm{keV}, 130 \mathrm{~mA}$ Hydrogen Ion Beam', these proceedings.

[4] D. Schrage, L. M. Young, W. Clark, T. Davis, F. Martinez, A. Naranjo and P. Roybal, 'A $6.7 \mathrm{MeV}$ CW RFQ Linac', these proceedings.

[5] J. D. Gilpatrick, J. F. Power, C. R. Rose, R. E. Shafer and R. B. Shurter, 'LEDA and APT Beam Diagnostics Instrumentation', these proceedings.

[6] J. E. Stovall, E. R. Gray, S. Nath, H. Takeda, R. L. Wood, L. M. Young, K. R. Crandall, 'Alignment and Steering Scenarios for the APT Linac', Proceedings of the XVIII International Linac Conference,, Geneva, Switzerland, August 26-30, 1996 p. 686.

[7] T. L. Owens, et.al., 'Phase Scan Signature Matching for Linac Tuning', Linac94 Proceedings, August 1994, Tsukuba, Japan, p893.

[8] J. T. Bradley, III, et. al., 'An Overview of the Low Energy Demonstration Accelerator (LEDA) Project RF Systems', these proceedings.

[9] S. Nath, E. R. Gray, T. P. Wangler and L. M. Young, 'Beam Dynamics Design for the APT Integrated Linac', these proceedings. 